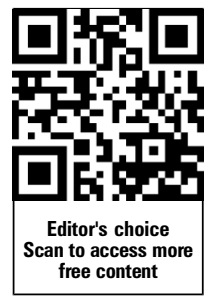

\title{
Prospective observational single-centre cohort study to evaluate the effectiveness of treating lupus nephritis with rituximab and mycophenolate mofetil but no oral steroids
}

\author{
Marie B Condon, ${ }^{1}$ Damien Ashby, ${ }^{1}$ Ruth J Pepper, ${ }^{1}$ H Terence Cook, ${ }^{1,2}$ Jeremy B Levy, \\ Megan Griffith, ${ }^{1}$ Tom D Cairns, ${ }^{1}$ Liz Lightstone ${ }^{1,2,3}$
}

\begin{abstract}
Handling editor Tore K Kvien
${ }^{1}$ Imperial College NHS

Healthcare Trust Lupus Centre, Hammersmith Hospital, London, UK

${ }^{2}$ Centre for Complement and Inflammation Research, Department of Medicine, Imperial College London, London, UK

${ }^{3}$ The Section of Renal and Vascular Inflammation Department of Medicine, Imperial College London, London, UK
\end{abstract}

\section{Correspondence to} Dr Liz Lightstone, Section of Renal and Vascular Inflammation, Department of Medicine, Imperial College London, Hammersmith Campus, Du Cane Road London W12 0NN, UK; I.lightstone@imperial.ac.uk

MG, TDC and LL contributed equally to the development of the entire project and are joint senior authors.

Accepted 14 April 2013 Published Online First 5 June 2013

\section{SLinked}

- http://dx.doi.org/10.1136/ annrheumdis-2013-203205

\section{ABSTRACT}

Objectives Lupus nephritis (LN) is a serious complication of systemic lupus erythematosus (SLE). All current treatment regimens include oral steroids, which are associated with severe adverse events and long-term damage. We have piloted a steroid-avoiding protocol (rituxilup) for the treatment of biopsy-proven active International Society of Nephrology/Renal Pathology Society (ISN/RPS) class III, IV, or class V LN.

Methods We report the findings from the first 50 consecutive patients, treated with 2 doses of rituximab $(1 \mathrm{~g})$ and methyl prednisolone $(500 \mathrm{mg})$ on days 1 and 15 , and maintenance treatment of mycophenolate mofetil. Patients on maintenance steroids or with lifethreatening SLE or requiring dialysis were excluded. Renal remission was defined as serum creatinine no greater than $15 \%$ above baseline; complete biochemical remission (CR) was defined as urine protein : creatinine ratio $(\mathrm{PCR})<50 \mathrm{mg} / \mathrm{mmol}$ or partial remission (PR) if PCR $>50 \mathrm{mg} / \mathrm{mmol}$ but non-nephrotic and $>50 \%$ reduction.

Results A total of $45(90 \%)$ patients achieved CR or PR by a median time of 37 weeks (range 4-200). Overall, $72 \%(n=36)$ achieved CR (median time 36 weeks $(11-58))$ and a further $18 \%(n=9)$ achieved persistent PR (median time 32 weeks (19-58)). By 52 weeks, CR and PR had been achieved in 52\% $(n=26)$ and 34\% $(n=17)$ respectively. In all, 12 relapses occurred in 11 patients, at a median time of 65.1 weeks (20-112) from remission. A total of $6 / 50$ patients had systemic flares. Of the 45 responders, only 2 required $>2$ weeks of oral steroids. Adverse events were infrequent; 18\% were admitted, $10 \%$ for an infective episode.

Conclusions The rituxilup cohort demonstrates that oral steroids can be safely avoided in the treatment of LN. If findings are confirmed, it could mark a step change in the approach to the treatment of $L N$.

\section{INTRODUCTION}

Nephritis is a serious complication of systemic lupus erythematosus (SLE) affecting up to $60 \%$ of adults with SLE. ${ }^{1}$ The current standard treatment for lupus nephritis (LN) is high dose corticosteroids (steroids) in combination with either cyclophosphamide (CyP) or mycophenolate mofetil (MMF). ${ }^{2}$ Recent randomised controlled trials show MMF to be equivalent to pulsed intravenous $\mathrm{CyP}^{3}{ }_{4}^{4}$ however, only prednisolone, aspirin and hydroxychloroquine are licensed for the treatment of $\mathrm{LN}$ in the UK. Steroids are common to all standard protocols. The relapsing/remitting course of LN means that patients routinely require long-term treatment. ${ }^{56}$ Chronic steroid exposure is associated with significant morbidity and premature mortality; monitoring and prophylaxis are required to reduce long-term complications. The resultant polypharmacy, in combination with steroid-induced cosmetic changes, may lead to non-adherence-a well-recognised cause of treatment failure in lupus. ${ }^{7-10}$ In general, decisions on dose and duration of steroid treatment tend to be influenced by the preferences of individual doctors rather than clinical parameters. ${ }^{11}$

In renal transplantation the use of induction regimens with depleting antibodies has allowed the safe introduction of steroid-free maintenance regimens. ${ }^{12}$ In systemic vasculitis, use of the $\mathrm{B}$ celldepleting monoclonal antibody, rituximab, has allowed reduction of concomitant immunosuppression. ${ }^{13} 14$ In LN we have previously reported that rituximab can be an effective treatment for $\mathrm{LN}$ in patients already on oral steroids and allows for a steroid dose reduction. ${ }^{15}$ Rituximab can be effective in refractory $\mathrm{LN}^{16-22}$ We report on the outcomes of the first 50 patients treated with our novel 'rituxilup' protocol, designed to avoid the use of maintenance oral steroids in LN. Patients who were not on maintenance oral steroids at the time of acute biopsy-proven LN were treated with two intravenous doses of rituximab and methylprednisolone and oral MMF alone.

\section{MATERIALS AND METHODS}

From 1 January 2006 until 1 November 2010, all patients at the Imperial College Healthcare NHS Trust Lupus Centre with biopsy-proven active International Society of Nephrology/Renal Pathology Society (ISN/RPS) class III, IV, or class V $\mathrm{LN}$, not taking long-term oral steroids, were treated with the rituxilup protocol. Patients were excluded if they had previously received rituximab, had life-threatening complications (eg, cerebritis), had a rapidly progressive glomerulonephritis, or required renal replacement treatment. All patients had at least 12 months of follow-up. Patients were 
treated with two doses of rituximab $1 \mathrm{~g}$ and methyl prednisolone $0.5 \mathrm{~g}$, on days 1 and 15 . Patients received MMF, initially $500 \mathrm{mg}$ twice a day, titrated (to a maximum dose of $1.5 \mathrm{~g}$ twice a day) to $12 \mathrm{~h}$ trough mycophenolic acid (MPA) levels of 1.2-2.4 mg/litre, providing leucocyte count and gastrointestinal symptoms allowed. Angiotensin receptor blockers and angiotensin-converting enzyme inhibitors were used in combination at the highest doses tolerated.

Complete biochemical remission (CR) was defined by the combination of a urine protein: creatinine ratio (PCR) of less than $50 \mathrm{mg}$ protein/mmol creatinine and serum creatinine no greater than $15 \%$ above baseline. Partial remission (PR) was defined by a urine PCR $<300 \mathrm{mg} / \mathrm{mmol}$, that is, non-nephrotic, with a $>50 \%$ reduction from baseline and serum creatinine no greater than $15 \%$ above baseline.

At 12 months, patients with a urine PCR $>50 \mathrm{mg} / \mathrm{mmol}$ and proteinuria that was not still falling underwent a further renal biopsy. Those with inactive disease on biopsy (as shown by the absence of active class III or IV lesions on light microscopy and the absence of new subepithelial deposits on electron microscopy (EM)) were defined as being in histological remission (HR) and were not offered augmentation of their immunosuppression. Relapse was defined as a persistent increase of $>30 \%$ in proteinuria and/or serum creatinine. B cell counts were measured by CD19 flow cytometry with B cell depletion defined as CD19 $<5$ cells $/ \mathrm{ml}$.

\section{STATISTICAL METHODS}

Continuous variables are expressed as median (IOR). Differences between groups and across first year timepoints were analysed using one-way analysis of variance (ANOVA) with Bonferroni corrected post hoc tests, using log transformation and geometric mean for skewed variables. Time to remission or relapse was estimated by the Kaplan-Meier method, with differences between groups assessed with the log rank test. Results with $p$ value less than 0.05 were considered significant. Statistical analyses were performed using SPSS V.16.0 (SPSS Inc., Chicago, Illinois, USA).

\section{RESULTS \\ Patient population}

A total of 50 consecutive patients were treated with the rituxilup protocol. Follow-up data were available on 49/50 with a median follow-up of 163 weeks (52-237). One patient was lost to follow-up after moving abroad.

The baseline characteristics of all patients are shown in table 1. The median age of the patients was 45 years. While a large proportion, $40 \%$, had active class IV or class IV+V LN, 22 (44\%) patients had pure class V LN. A significant proportion (58\%) had extrarenal involvement at presentation.

\section{Baseline biochemistry}

Severity varied according to renal histology with higher mean serum creatinine, lower mean serum albumin and higher mean urine PCR seen in patients with class IV LN compared with those with class III or V (figure 1). The cohort had severe LN at baseline with median (IOR) values for serum albumin of $24 \mathrm{~g} /$ litre (18-31) serum creatinine $92 \mathrm{mmol} /$ litre (78-130) and urine PCR $422 \mathrm{mg} / \mathrm{mmol}$ (175-894) (figure 2).

\section{Remission induction}

By the data cut-off point of 1 November 2010, CR or PR had been achieved in $45 / 50$ patients (90\%) at median time of 37 weeks (4-200), (figure 3A). Overall, CR was achieved in 36 patients (72\%) at median time of 36 weeks (11-58) (figure 3A), with their latest median creatinine being $70 \mathrm{mmol} / \mathrm{litre}$ (63-106). Persistent PR was achieved in nine patients (18\%) at median time of 32 weeks (19-58). Their median creatinine by latest follow-up was $76 \mu \mathrm{mol} /$ litre (64-200) and median PCR of $174 \mathrm{mg} / \mathrm{mmol}$ (68-408). By 26 weeks 16/50 (32\%) had achieved a CR and $15 / 50(30 \%)$ had achieved a PR. By 1 year this had increased to $26 / 50(52 \%)$ in CR and $17 / 50$ (34\%) in PR (figure $3 \mathrm{~A})$. The time to $\mathrm{CR}$ or PR was not influenced by class of $\mathrm{LN}$ at baseline (figure $3 \mathrm{~B}$ ).

There were 24 patients who had not achieved a PCR $<50 \mathrm{mg} / \mathrm{mmol}$ by 52 weeks. Of 24,4 had a falling PCR $(2 / 4$ subsequently reached CR by data cut-off point) and $5 / 24$ had or were being switched to alternative treatment regimens $(2 / 5$ had relapsed from $P R$ and $3 / 5$ had failed to respond ( $2 / 3$ before 12 months and $1 / 3$ at 16 months)). There were therefore 15 patients eligible for rebiopsy under the regimen protocol. Out of 15,2 failed to attend. Repeat biopsies in 8/15 showed HR (6/8 subsequently progressing to CR by data cut-off point) and in $4 / 15$ showed active disease ( $3 / 4$ were redosed with rituximab (1/3 went into $C R$ ) and $1 / 4$ had her MMF dose optimised (achieved CR before later relapse)). A further patient (1/15) achieved HR but had diabetic change on repeat biopsy which accounted for the persistent proteinuria.

Overall, there was no significant change in mean serum creatinine. For those with baseline serum creatinine $<110 \mu \mathrm{mol} /$ litre, the median (IOR) creatinine at baseline was $80 \mathrm{mmol} /$ litre (73-87) falling to $73 \mathrm{mmol} /$ litre $(66-87)$ at 1 year and $68 \mathrm{mmol} /$ litre $(63-73)$ by 3 years. Of the 20 patients with baseline serum creatinine $>110 \mathrm{mmol} /$ litre, 18 (90\%) showed improvement over time falling from a baseline median of $146 \mathrm{mmol} /$ litre (126-191) to $125 \mathrm{mmol} /$ litre (76-164) at 1 year and $133 \mathrm{mmol} /$ litre $(105-171)$ at 3 years. Only six patients overall had a rise of $>15 \%$ above baseline at latest follow-up, three of whom initially achieved PR and then relapsed.

\section{Renal relapses}

There were 12 relapses in 11 patients (22\%), all nephrotic. The median time to relapse from remission was 65.1 weeks (20-112). Of these, eight relapses (in seven patients) occurred from CR and four from PR. One patient, with class V at baseline, did not have a repeat biopsy as relapse occurred in the context of severe systemic infection secondary to a diverticular abscess and the patient was not fit for further immunosuppression. In 4/11 patients, the baseline and relapse biopsies showed class $\mathrm{V}$ LN. Of these, one patient relapsed twice soon after B cell repletion - this was in the context of MMF having been withdrawn due to recurrent persistent upper respiratory tract infections. Of the $6 / 11$ with class IV LN at baseline, repeat biopsy demonstrated active class IV nephritis in $3 / 6$, class III with fibrinoid necrosis and cellular crescents in $1 / 6$ (she subsequently was found to have myeloperoxidase (MPO)-positive anti-neutrophil cytoplasmic antibody (ANCA) vasculitis with pulmonary haemorrhage), class $\mathrm{V}$ in 1 and 1 declined biopsy.

Seven relapses (in six patients) were treated with further rituximab (two doses of $1 \mathrm{~g}$ with or without methylprednisolone) with three achieving CR. One achieved PR and three failed to respond.

\section{Systemic flares of SLE and use of oral steroids}

Of the 50 patients, 6 had systemic flares, mostly mild. One patient had skin involvement that resolved following an increase in MMF from $750 \mathrm{mg}$ twice a day to $1 \mathrm{~g}$ twice a day. One patient developed thrombocytopoenia and neutropoenia, 
Table 1 Baseline demographics of rituxilup cohort

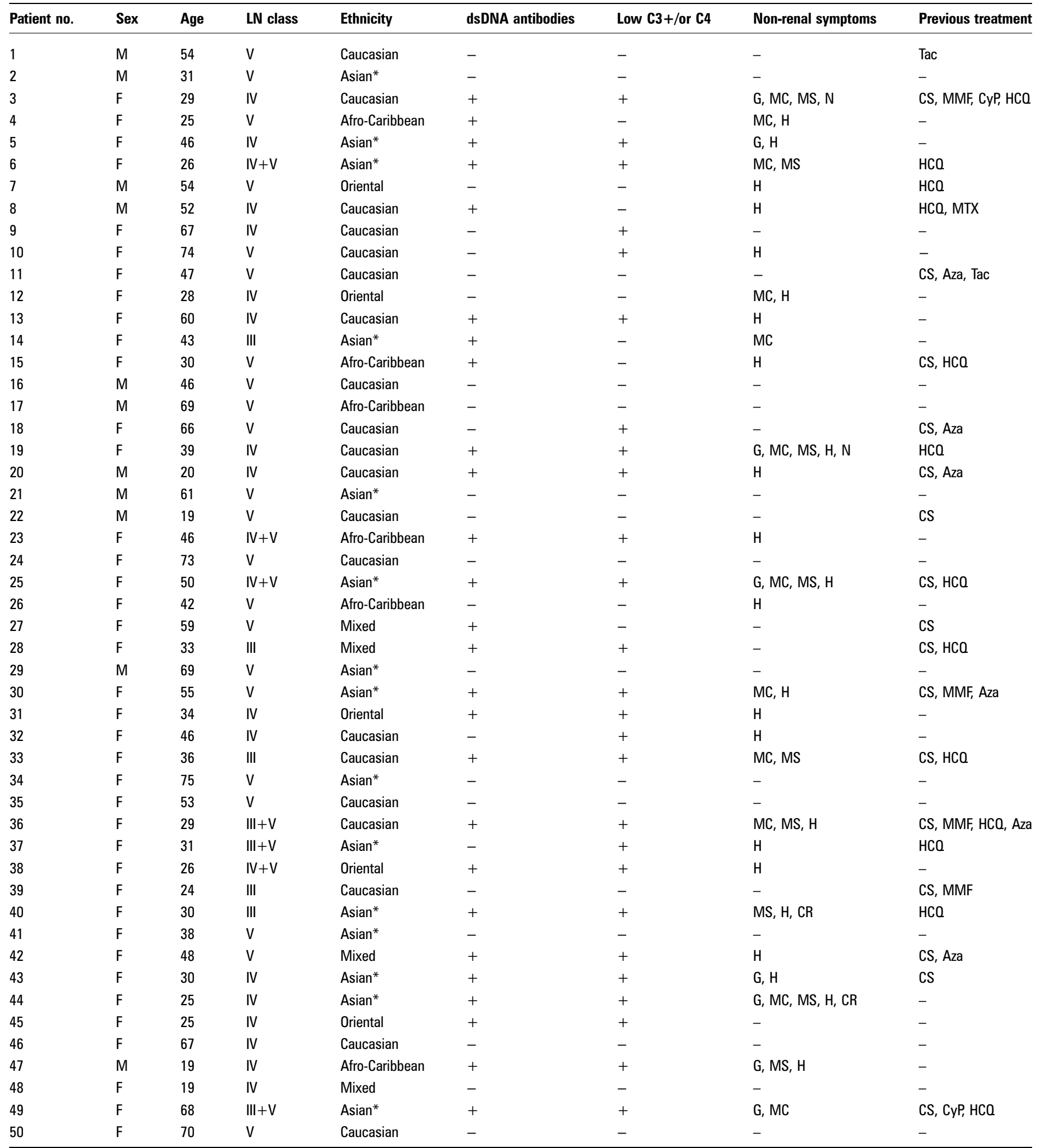

${ }^{*}$ Asian denotes individuals from Indian subcontinent.

Double-stranded DNA antibody (dsDNA Ab) quantitative assay: negative $<30 \mu / \mathrm{ml}$ : Low C3 $<0.7 \mathrm{~g} /$ litre; Low C4 $<0.16 \mathrm{~g} /$ litre

Non-renal symptoms (as described in the British Isles Lupus Assessment Group (BILAG) Index ${ }^{23}$ ): CR, cardiorespiratory; G, general; $\mathrm{H}$, haematological; MC, mucocutaneous;

MS, musculoskeletal; N, neurological.

Previous treatments: Aza, azathioprine; CS, corticosteroids; CyP, cyclophosphamide; HCQ, hydroxychloroquine; LN, lupus nephritis; MMF, mycophenolate mofetil; MTX, methotrexate; Tac, tacrolimus.

which responded to redosing with methyl prednisolone and rituximab. Two patients required a single short course of prednisolone ( $<2$ weeks) for systemic symptoms with a maximum dose of $30 \mathrm{mg}$. Only two patients who stayed on the rituxilup protocol required maintenance steroids: one patient required long-term oral steroid treatment for severe systemic symptoms (including tongue swelling and serositis) at the time of biopsy, tapering from $30 \mathrm{mg}$ once a day to $5 \mathrm{mg}$ once a day. One 


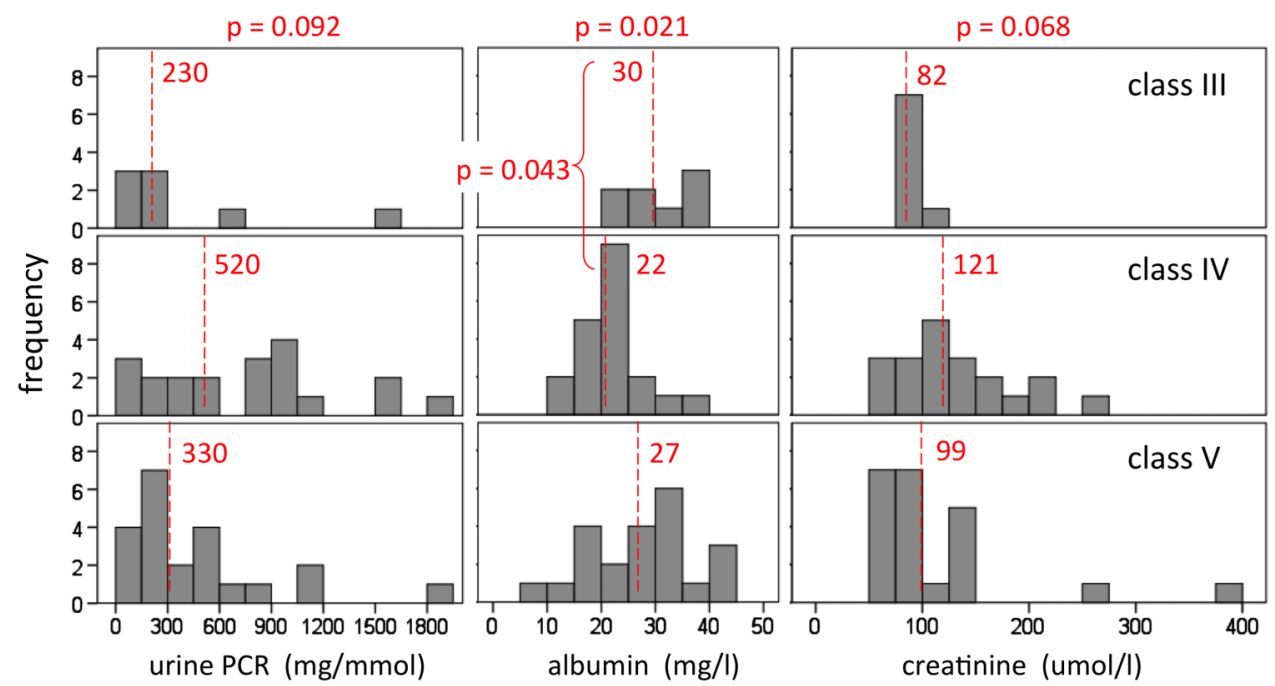

Figure 1 Baseline biochemistry by histological class. Distributions of baseline urine protein : creatinine ratio (PCR), serum albumin and creatinine according to histological class with mean/geometric mean parameter values. $p$ Values for the existence of a class effect on each parameter are given at the top, with the only significant post hoc difference given within the chart. This figure is only reproduced in colour in the online version.

patient had three short courses of prednisolone for flares of cutaneous lupus.

\section{Depletion of B cells}

Complete data were available for 42/50 patients, of whom 39/ $42(93 \%)$ showed depletion to $<5$ B lymphocytes/ml following

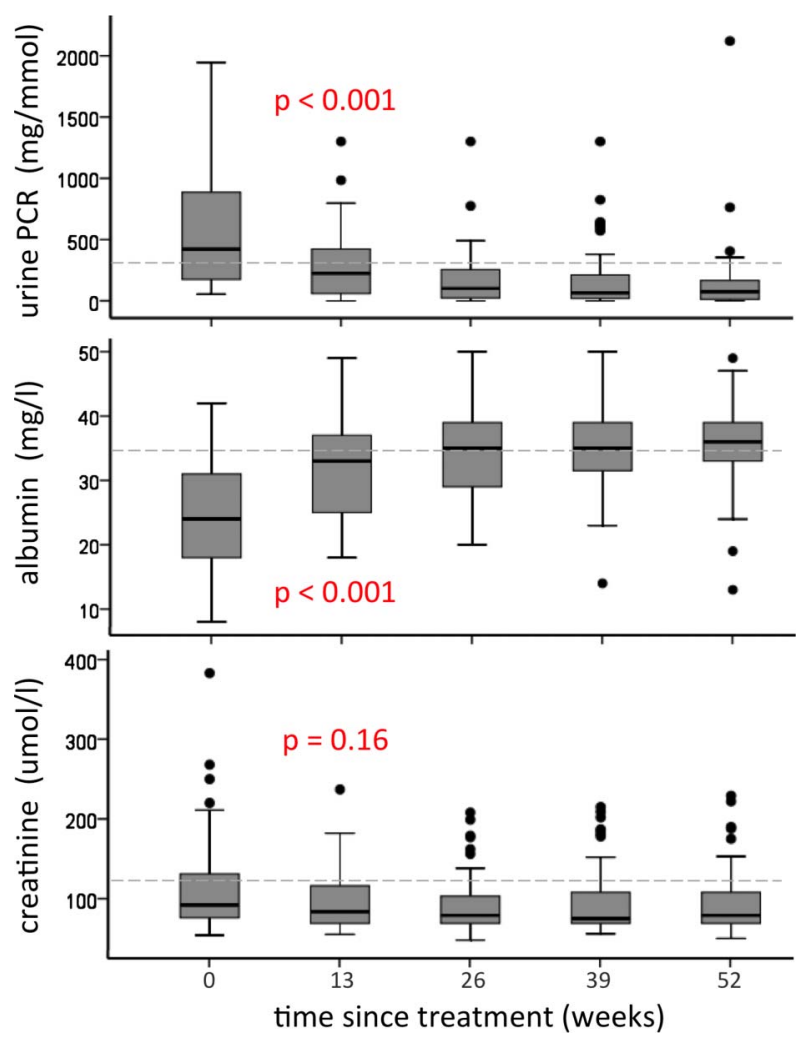

Figure 2 Change in renal function during the first year. Box and whisker distribution of renal function parameters during the first year after treatment, with $p$ values for the existence of a timepoint effect. Mean urine protein:creatinine ratio and serum albumin are significantly different from baseline at every follow-up timepoint $(p<0.001$ for all comparisons, with Bonferroni correction). This figure is only reproduced in colour in the online version. rituximab treatment. Of the three patients who did not fully deplete (nadir counts of 9,5 and 35 B lymphocytes/ml respectively) two achieved CR (at 73 weeks and 59 weeks respectively) and one was a non-responder.

\section{Severe adverse events}

18 per cent (9/50) of patients had an adverse event involving an inpatient stay, $5 / 9$ were due to an infective episode. One patient developed shingles (table 2 ).

One patient (2\%) developed type 2 diabetes following induction treatment with rituximab and methyl prednisolone.

Two patients died (4\%). A 61-year-old patient died 31 months after rituximab treatment. She was in CR at the time of death and died from the complications of surgery for severe peripheral vascular disease. The second was a 71-year-old man who died suddenly at home 28 months after first treatment with rituximab. At 1 year he was in PR and was rebiopsied, redosed with rituximab at 18 months and was in $P R$ at the time of death.

\section{DISCUSSION}

We have previously reported the use of rituximab and MMF for the treatment of patients with acute LN already on oral steroids and demonstrated a steroid sparing effect. ${ }^{15}$ The use of rituximab and methyl prednisolone, in the absence of any other immunosuppression, was reported to be effective in the treatment of flares of proliferative $\mathrm{LN}$ in a series of three patients. ${ }^{24}$ Using a steroid sparing regimen, based on our rituxilup regimen, in a group of patients with new onset non-renal lupus, Ezeonyeji and colleagues demonstrated this regimen to be safe, effective and to reduce the overall steroid burden in SLE, when assessed by the British Isles Lupus Assessment Group (BILAG) disease activity index. ${ }^{25}$ Our study is the first report of a sizeable cohort of patients with $\mathrm{LN}$ treated with a rituximab-based regimen (the rituxilup protocol) that avoids the use of any oral steroids. The rituxilup protocol was well tolerated and achieved high rates of renal remission. Although this was a prospective observational study, not a clinical trial, the rates of CR $32 \%$ and CR or PR (62\%) at 26 weeks compare favourably with those achieved by patients treated with steroids and MMF in the Aspreva Lupus Management Study 


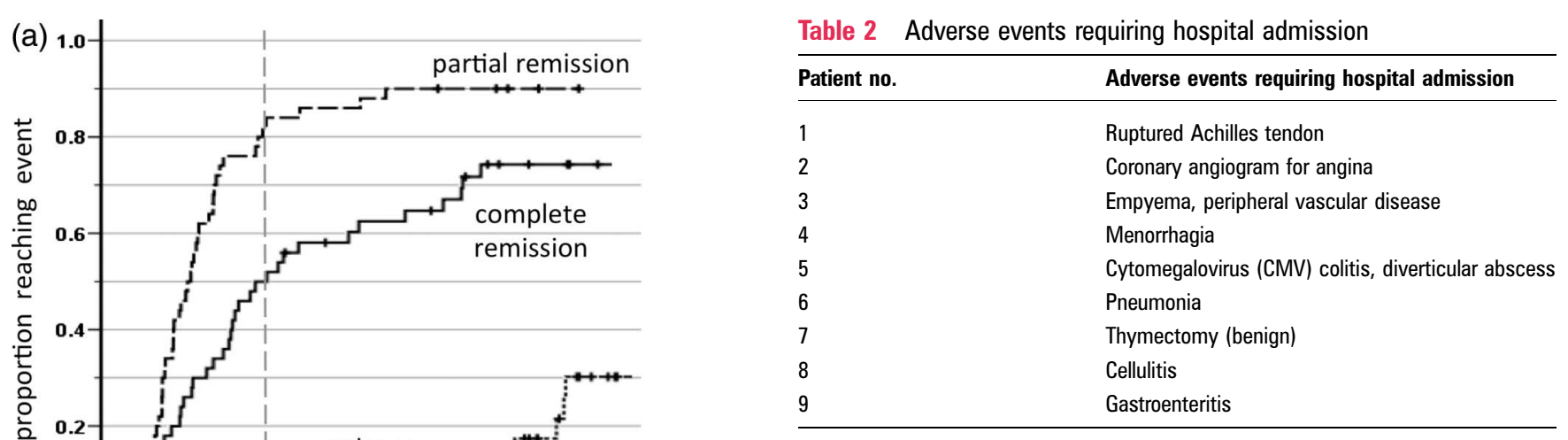

long-term chronic histological damage. It is noteworthy that 8/ 19 patients who had achieved a stable PR (based on persistent proteinuria with a PCR $>50 \mathrm{mg} / \mathrm{mmol}$ ) had repeat biopsies demonstrating HR on the basis of absence of active lesions and no new subepithelial deposits on EM. Six of the eight went on to achieve CR at a median time of 26 weeks after their repeat biopsy without a change in treatment thus demonstrating that HR was an excellent predictor of a good outcome.

The high proportion of patients achieving remission at 6 months also suggests a favourable long-term renal outcome for this group of patients, and importantly $50 \%$ of our cohort achieved a PCR of $<100 \mathrm{mg} / \mathrm{mmol}$ by 6 months. Houssiau et $\left.a\right|^{27}$ recently reported the long-term follow-up of the Eurolupus trial and confirmed that an early (3-6 months) response (reduction in proteinuria to $<1 \mathrm{~g} / 24 \mathrm{~h}$ ) predicted a good long-term outcome (creatinine $<1.4 \mathrm{~g} / \mathrm{dl}$ at 10 years).

A total of $44 \%$ of our patients, of whom $50 \%$ were nephrotic at presentation, had pure class $\mathrm{V}$ lesions at baseline. The combination of rituximab and cyclophosphamide, but with steroids, has been shown to be effective in refractory class V LN. ${ }^{28}{ }^{29}$ In an analysis of outcomes of the patients with pure class V LN in the US and ALMS trials, Radhakrishnan et a ${ }^{30}$ demonstrated no differences in outcomes at 24 weeks whether patients were treated with MMF or cyclophosphamide. Of those treated with MMF and steroids whom had nephrotic class $\mathrm{V}$ at presentation, CR at 24 weeks was achieved in none of those in the US trial, and just $7 \%$ of those in the ALMS trial. In contrast, in our cohort, $18 \%$ of the nephrotic class $\mathrm{V}$ patients achieved CR at 6 months, rising to $36 \%$ by 1 year. Of the total group of 22 class $\mathrm{V}$ patients, at 6 months $32 \%$ were in CR, $23 \%$ in PR and by 12 months the proportions had risen to $38 \%$ and $24 \%$ respectively.

Another striking feature of our study was the low rate of systemic features during treatment. This may reflect patients being treated relatively early in the course of their disease. This is analogous to the growing evidence in rheumatoid arthritis where early use of biological agents is associated with better long-term outcomes and reduced damage. ${ }^{31}$ It is also possible that titrating MMF dosing to trough blood MPA levels, may have reduced or successfully treated systemic relapses. Other authors have reported the efficacy of MMF in treating extrarenal disease in lupus though have queried whether responses are maintained beyond 2 years. ${ }^{32}{ }^{33}$ Hydroxychloroquine was also a useful adjunct.

Clearly, a prospective observational study such as this cannot define the role of rituximab in treating LN. Rituximab is a very attractive therapeutic agent for lupus because it has a very long safety record from its extensive use in non-Hodgkin's lymphoma and because it has a biologically relevant target namely CD20 expressed on B cells from pre-B cells up to, but not including, lent renal function in the majority of patients suggest that the possibly slower rates of response were not at the expense of 
plasma cells. Very recent data from Vital et al have demonstrated that clinical responses to rituximab in patients with lupus are strongly correlated with the degree, duration and specific B cell phenotypes that are depleted and repopulate. ${ }^{34}$

There is currently a mismatch between the perceived value of rituximab for the treatment of $\mathrm{LN}$ and the published data. The only randomised controlled trial of rituximab in $\mathrm{LN}$, the 'LUpus Nephritis Assessment with Rituximab' (LUNAR) trial, comparing $\mathrm{MMF}$ + steroids + placebo with $\mathrm{MMF}$ + steroids + rituximab in 144 patients with class III or class IV LN, failed to achieve the primary end point of superiority in the proportion of patients in CR or PR at 52 weeks in the rituximab treated arm. $^{35}$ At 1 year there was no difference in the proportion achieving CR but a $15 \%$ increase in those achieving a PR in the rituximab group. LUNAR was underpowered to detect the significance of the increase in PR rates seen. ${ }^{36}$ Another problem with LUNAR and other observational studies is the continued reliance on long-term oral steroids. In the LUNAR study the data suggest that patients were failing to have their steroids cut according to planned taper, highlighting how difficult it is to wean steroids once started. This may reflect treatment for extrarenal manifestations but it is likely that clinicians were reluctant to cut steroids in the face of ongoing proteinuria, and presumed disease activity in the absence of repeat biopsies.

No trial has compared rituximab replacing a traditional agent with standard treatment. There is little argument that MMF plus steroids or intravenous cyclophosphamide plus steroids are effective combinations in the treatment of LN. Our study suggests that the combination of rituximab, low dose intravenous methyl prednisolone and MMF without oral steroids is also an effective regimen. The avoidance of steroids has huge potential benefits in this group of patients, particularly as they often require immunosuppression for many years, they may avoid the unpleasant cosmetic effects of steroids (with the associated psychological damage) and the long-term damage attributable to steroids.

This study marks a step change in the approach to the treatment of LN. For the first time in 60 years, it has suggested that oral steroids can be safely avoided in the treatment of LN without apparent reduction in efficacy or increase in relapse rates, at least up to 3 years of follow-up. The protocol will now be formally assessed in a prospective multicentre international randomised controlled trial (The Rituxilup Trial NCT01773616) and for the first time may offer a realistic possibility of discarding arguably the most toxic element in standard long-term treatment of this challenging disease.

Correction notice This article has been corrected since it was published Online First. The contributors statement has been added on the first page.

Acknowledgements We are grateful for funding from the NIHR Imperial College Biomedical Research Centre. We thank Dr Chris Kirwan who helped establish the original database and Dr Janet Lee and colleagues in the Lesley Brent laboratory at the ICHNT Renal and Transplant Centre who provide excellent technical support with the MPA levels.

Contributors TDC, MG, JBL and LL conceived and developed the protocol, and were responsible for the clinical care of the patients as well as analysing the data and drafting of the report. MBC and RP collated all the data and MBC helped to analyse the data and draft the report. DA performed the statistical analysis. HTC performed the histological analysis.

Competing interests LL: honoraria to attend meetings/or for contributions to advisory boards from GlaxoSmithKline, Roche, Aspreva Pharmaceuticals, Biogen Idec, Vifor and Genentech (all less than f2000). MG: honoraria to attend meetings from GlaxoSmithKline.

Ethics approval This novel protocol was designed and agreed by the Glomerulonephritis Clinical Research Group at Imperial College Healthcare NHS Trust. All elements of the protocol had been used previously for the treatment of lupus nephritis, however not in this combination. This was adopted at the outset of the cohort as our standard treatment protocol for all patients with lupus nephritis who were not currently taking oral steroids and who did not have cerebral or other life-threatening forms of lupus. It was not an experimental protocol, but was our standard clinical protocol and in discussion with the Chair of the local Research Ethics Committee it was agreed that therefore Ethics approval was not required.

Provenance and peer review Not commissioned; externally peer reviewed.

\section{REFERENCES}

1. Patel M, Clarke AM, Bruce IN, et al. The prevalence and incidence of biopsy-proven lupus nephritis in the UK: evidence of an ethnic gradient. Arthritis Rheum 2006;54:2963-9.

2. Lightstone L. Lupus nephritis: where are we now? Curr Opin Rheumatol 2010;22:252-6.

3. Appel GB, Contreras G, Dooley MA, et al. Mycophenolate mofetil versus cyclophosphamide for induction treatment of lupus nephritis. J Am Soc Nephrol 2009;20:1103-12.

4. Ginzler EM, Dooley MA, Aranow C, et al. Mycophenolate mofetil or intravenous cyclophosphamide for lupus nephritis. N Engl J Med 2005;353:2219-28.

5. Houssiau FA, D'Cruz D, Sangle $S$, et al. Azathioprine versus mycophenolate mofetil for long-term immunosuppression in lupus nephritis: results from the MAINTAIN Nephritis Trial. Ann Rheum Dis 2010;69:2083-9.

6. Dooley MA, Jayne D, Ginzler EM, et al. Mycophenolate versus azathioprine as maintenance therapy for lupus nephritis. N Engl J Med 2011;365:1886-95

7. Gladman DD, Urowitz MB, Rahman $\mathrm{P}$, et al. Accrual of organ damage over time in patients with systemic lupus erythematosus. J Rheumatol 2003;30:1955-9.

8. Zonana-Nacach A, Barr SG, Magder LS, et al. Damage in systemic lupus erythematosus and its association with corticosteroids. Arthritis Rheum 2000;43:1801-8

9. Masood S, Jayne D, Karim Y. Beyond immunosuppression — challenges in the clinical management of lupus nephritis. Lupus 2009;18:106-15.

10. Suleiman AB, Morad Z, Tong CT. Mortality in SLE nephritis. Ann Acad Med Singapore 1987;16:175-8.

11. Walsh M, Jayne D, Moist L, et al. Practice pattern variation in oral glucocorticoid therapy after the induction of response in proliferative lupus nephritis. Lupus 2010;19:628-33

12. Borrows R, Loucaidou M, Van Tromp J, et al. Steroid sparing in renal transplantation with tacrolimus and mycophenolate mofetil: three-year results. Transplant Proc 2005;37:1792-4.

13. Mansfield N, Hamour S, Habib AM, et al. Prolonged disease-free remission following rituximab and low-dose cyclophosphamide therapy for renal ANCA-associated vasculitis. Nephrol Dial Transplant 2011;26:3280-6.

14. Stone JH, Merkel PA, Spiera R, et al. Rituximab versus cyclophosphamide for ANCA-associated vasculitis. N Engl J Med 2010;363:221-32.

15. Pepper R, Griffith M, Kirwan C, et al. Rituximab is an effective treatment for lupus nephritis and allows a reduction in maintenance steroids. Nephrol Dial Transplant 2009;24:3717-23.

16. Fra GP, Avanzi GC, Bartoli E. Remission of refractory lupus nephritis with a protocol including rituximab. Lupus [Case Reports] 2003;12:783-7.

17. van Vollenhoven RF, Gunnarsson I, Welin-Henriksson E, et al. Biopsy-verified response of severe lupus nephritis to treatment with rituximab lanti-CD20 monoclonal antibody) plus cyclophosphamide after biopsy-documented failure to respond to cyclophosphamide alone. Scand J Rheumatol 2004;33:423-7.

18. Edelbauer M, Jungraithmayr T, Zimmerhackl LB. Rituximab in childhood systemic lupus erythematosus refractory to conventional immunosuppression: case report. Pediatr Nephrol 2005;20:811-13.

19. Leandro MJ, Cambridge G, Edwards JC, et al. B-cell depletion in the treatment of patients with systemic lupus erythematosus: a longitudinal analysis of 24 patients. Rheumatology (Oxford) 2005;44:1542-5.

20. Sfikakis PP, Boletis JN, Lionaki S, et al. Remission of proliferative lupus nephritis following B cell depletion therapy is preceded by down-regulation of the T cell costimulatory molecule CD40 ligand: an open-label trial. Arthritis Rheum 2005; 52:501-13.

21. Van den Bergh B, Selleslag D, Boelaert JR, et al. Management of therapy-resistant systemic lupus erythematosus with rituximab: report of a case and review of the literature. Acta Clin Belg 2005;60:102-5.

22. Jacobson SH, van Vollenhoven R, Gunnarsson I. Rituximab-induced long-term remission of membranous lupus nephritis. Nephrol Dial Transplant 2006;21:1742-3.

23. Hay EM, Bacon PA, Gordon C, et al. The BILAG index: a reliable and valid instrument for measuring clinical disease activity in systemic lupus erythematosus. Q J Med 1993;86:447-58.

24. Moroni G, Gallelli B, Banfi G, et al. Rituximab monotherapy for remission induction of proliferative lupus nephritis flares: description of 3 cases. J Nephrol 2010;23:357-61.

25. Ezeonyeji AN, Isenberg DA. Early treatment with rituximab in newly diagnosed systemic lupus erythematosus patients: a steroid-sparing regimen. Rheumatology (Oxford) 2012;51:476-81. 
26. Chan TM, Tse KC, Tang CS, et al. Long-term study of mycophenolate mofetil as continuous induction and maintenance treatment for diffuse proliferative lupus nephritis. J Am Soc Nephrol 2005;16:1076-84.

27. Houssiau FA, Vasconcelos C, D'Cruz D, et al. The 10-year follow-up data of the Euro-Lupus Nephritis Trial comparing low-dose and high-dose intravenous cyclophosphamide. Ann Rheum Dis 2010;69:61-4.

28. Jonsdottir T, Sundelin B, Welin Henriksson E, et al. Rituximab-treated membranous lupus nephritis: clinical outcome and effects on electron dense deposits. Ann Rheum Dis 2011;70:1172-3.

29. Jonsdottir T, Gunnarsson I, Mourao AF, et al. Clinical improvements in proliferative vs membranous lupus nephritis following B-cell depletion: pooled data from two cohorts. Rheumatology (Oxford) 2010;49:1502-4.

30. Radhakrishnan J, Moutzouris DA, Ginzler EM, et al. Mycophenolate mofetil and intravenous cyclophosphamide are similar as induction therapy for class $\mathrm{V}$ lupus nephritis. Kidney Int 2010;77:152-60.
31. Venkateshan SP, Sidhu S, Malhotra S, et al. Efficacy of biologicals in the treatment of rheumatoid arthritis. a meta-analysis. Pharmacology 2009;83:1-9.

32. Posalski JD, Ishimori M, Wallace DJ, et al. Does mycophenolate mofetil prevent extra-renal flares in systemic lupus erythematosus? Results from an observational study of patients in a single practice treated for up to 5 years. Lupus 2009;18:516-21.

33. Karim MY, Alba P, Cuadrado MJ, et al. Mycophenolate mofetil for systemic lupus erythematosus refractory to other immunosuppressive agents. Rheumatology (Oxford) 2002;41:876-82

34. Vital EM, Dass S, Buch MH, et al. B cell biomarkers of rituximab responses in systemic lupus erythematosus. Arthritis Rheum 2011:63:3038-47.

35. Rovin $\mathbf{B H}$, Furie $\mathrm{R}$, Latinis $\mathrm{K}$, et al. Efficacy and safety of rituximab in patients with active proliferative lupus nephritis: The Lupus Nephritis Assessment with Rituximab study. Arthritis Rheum 2012;64:1215-26.

36. Lightstone L. The landscape after LUNAR: Rituximab's crater-filled path. Arthritis Rheum 2012;64:962-5. 